\title{
Transcription at the edge
}

Chromosome positioning is thought to expose genomic loci to functionally distinct regions of the nucleus, generating transcriptional regulatory domains that favour either activation or repression. For example, components of the nuclear envelope, including the nuclear pore complex (NPC), were originally thought to assert a repressive role in

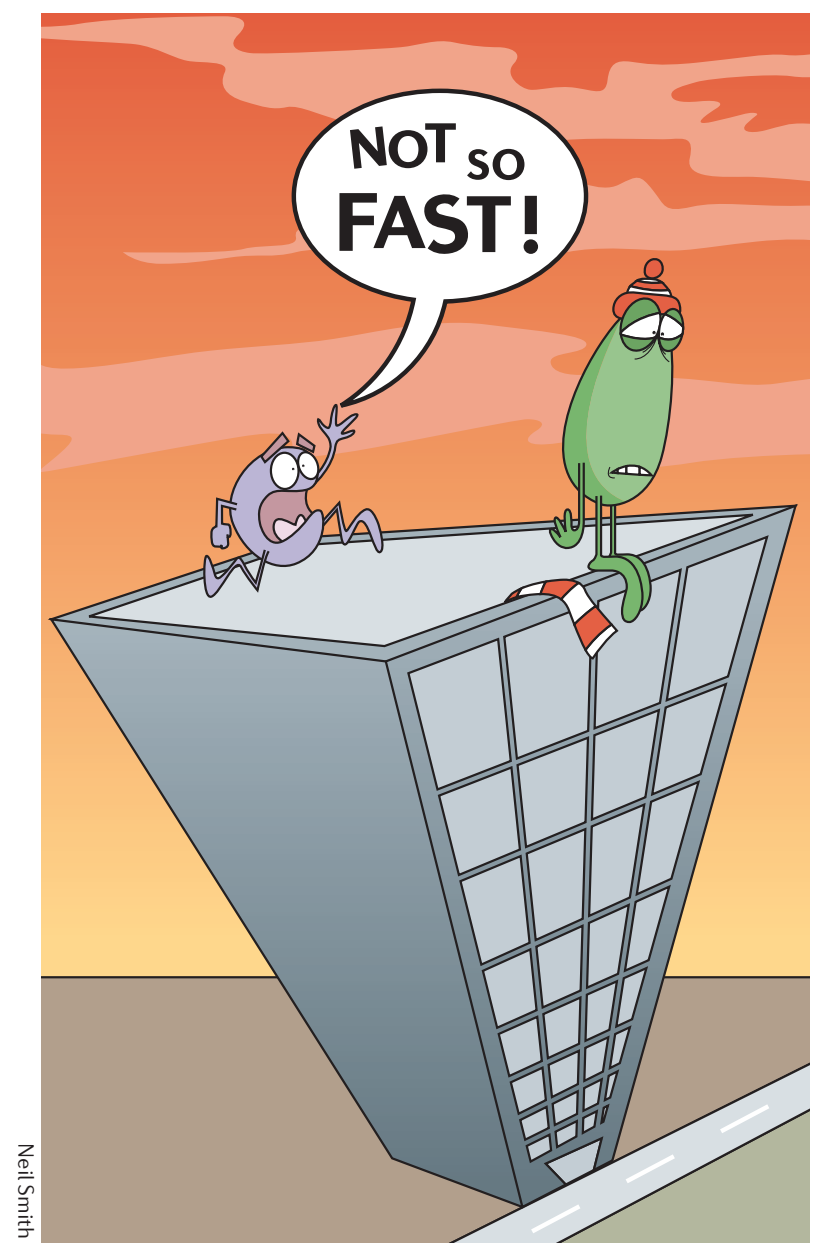

transcription. However, this mode of regulation seems to be complex; recent studies in Saccharomyces cerevisiae and Drosophila melanogaster have shown that populations of both active and silent genes interact with components of the nuclear envelope. But what happens in human cells?

Pamela Silver and colleagues investigated the relationship between the mammalian NPC and the human genome by generating high-resolution, chromosome-wide binding maps of human nucleoporin-93 (NUP93). Consistent with previous findings in yeast and flies, they found that the NPC interacts with chromatin. In human cells, NUP93 associates with regions of transcriptional repression and enriched heterochromatin on chromosomes 5, 7 and 16.

To probe the functionality of this association, Silver and co-workers analysed the effects of global histone acetylation on NPC-chromatin interactions by treating cells with Trichostatin-A (TSA), a reversible histone deacetylase inhibitor. Treating cells with TSA changed the genomic organization significantly and NUP93 distribution was altered across all three chromosomes. Analysis of NUP93-binding sites in TSA-treated cells indicated that regions associated with increased transcriptional activity become associated with NUP93. These include promoter regions and transcription start sites, specific histone methylations that are associated with active genes and RNA polymerase II-associated regions, and euchromatin.

Taken together, these findings indicate that global histone acetylation results in a genome-wide reorganization event that exchanges silent for active chromatin at NPCs and point to a role for the mammalian nuclear transport machinery in transcriptional regulation. In yeast, NPC association can increase mRNA processing and export, regulate the levels of gene expression and establish an epigenetic state that confers transcriptional memory and rapid reactivation of genes. Whether the mammalian NPC functions similarly to its yeast counterpart remains to be seen.

Ekat Kritikou, Senior Editor, Nature Reviews Molecular Cell Biology

ORIGINAL RESEARCH PAPER Brown, C. R. et al. Global histone acetylation induces functional genomic reorganization at mammalian nuclear pore complexes. Genes Dev. 22, 627-639 (2008) FURTHER READING Lanctôt, C. et al. Dynamic genome architecture in the nuclear space: regulation of gene expression in three dimensions. Nature Rev. Genet. 8, 104-115 (2007) | Akhtar, A. \& Gasser, S. M. The nuclear envelope and transcriptional control. Nature Rev. Genet. 8, 507-517 (2007) 